\section{(1) \\ CrossMark}

\title{
Effects of the "Living well with COPD" intervention in primary care: a comparative study
}

\author{
Claudia Steurer-Stey ${ }^{1,2,3}$, Kaba Dalla Lana ${ }^{1,2,3}$, Julia Braun², Gerben ter Riet ${ }^{4}$ \\ and Milo A. Puhan ${ }^{2}$
}

Affiliations: ${ }^{1}$ Institute of Primary Care, University of Zurich, Zurich, Switzerland. ${ }^{2}$ Epidemiology, Biostatistics and Prevention Institute, University of Zurich, Zurich, Switzerland. ${ }^{3}$ mediX Group Practice, Zurich, Switzerland. ${ }^{4}$ Dept of General Practice, Academic Medical Center, University of Amsterdam, Amsterdam, The Netherlands.

Correspondence: Claudia Steurer-Stey, Epidemiology, Biostatistics and Prevention Institute, University of Zurich, Hirschengraben 84, 8001 Zurich, Switzerland. E-mail: claudia.steurer-stey@uzh.ch

@ERSpublications

COPD self-management coaching improves health-status and lowers exacerbation rates among patients in primary care http://ow.ly/jZPw30gMbwB

Cite this article as: Steurer-Stey C, Dalla Lana K, Braun J, et al. Effects of the "Living well with COPD" intervention in primary care: a comparative study. Eur Respir J 2018; 51: 1701375 [https://doi.org/10.1183/ 13993003.01375-2017].

ABSTRACT The pivotal objective of chronic obstructive pulmonary disease (COPD) self-management programmes is behaviour change to avoid moderate and severe exacerbations and improve health related quality of life.

In a prospectively planned, controlled study, COPD patients who participated in the "Living well with COPD" (LWWCOPD) self-management intervention were compared with usual care patients from the primary care COPD Cohort ICE COLD ERIC, who did not receive self-management intervention (NCT00706602) The primary outcome was behaviour change and disease-specific health related quality of life after 1 year. Secondary end-points included exacerbation rates. We calculated mixed linear, zeroinflated negative binomial and logistic regression models and used propensity scores to counteract confounding.

467 patients, 71 from the LWWCOPD and 396 from the usual care cohort, were included. The differences between intervention and control were 0.54 (95\% CI 0.13-0.94) on the Chronic Respiratory Questionnaire domain "mastery", 0.55 (95\% CI 0.11-0.99) on "fatigue", 0.54 (0.14-0.93) on "emotional function" and 0.64 (95\% CI $0.14-1.14)$ on "dyspnoea". The intervention considerably reduced the risk of moderate and severe exacerbations (incidence rate ratio 0.36 , 95\% CI $0.25-0.52$ ).

Self-management coaching in primary care improves health-related quality of life and lowers exacerbation rates and health care use.

Received: July 092017 | Accepted after revision: Sept 282017

Support statement: AstraZeneca Switzerland provided an unrestricted grant for chronic care and patient education. The ICE COLD ERIC cohort project was supported by the Swiss National Science Foundation (grant \# 3233B0/115216/1), the Dutch Asthma Foundation (grant \# 3.4.07.045) and the Zurich Lung Association (unrestricted grants). Funding information for this article has been deposited with the Crossref Funder Registry.

Conflict of interest: Disclosures can be found alongside this article at erj.ersjournals.com

Copyright @ERS 2018 


\section{Introduction}

A patient-centred approach to the care of patients with chronic conditions, such as chronic obstructive pulmonary disease (COPD), is long overdue. This approach emphasises patient counselling and self-management as key components of successful disease management [1-3]. However, current care practices for COPD are mainly reactive to acute exacerbations of COPD. Instead, care should be proactive, coordinated and supportive; patients should be encouraged to acquire and apply self-management skills on a daily basis [3]. Often, providing self-management education is equated with conveying information about the disease, despite strong evidence that this traditional approach is insufficient [4,5]. The key objective of self-management interventions is to change behaviour [6-8].

The Canadian disease management programme, "Living well with COPD" (LWWCOPD) (www. livingwellwithcopd.com), was probably the first COPD self-management programme based on a leading model of chronic disease management, known as the Chronic Care Model [1]. Recently, Benzo et al. [9] tested a health coaching intervention aimed at empowering patients and changing behaviour after a severe exacerbation that required hospital admission. This intervention reduced rehospitalisation due to acute exacerbation of COPD for 1, 3 and 6 months, and improved health-related quality of life (HRQoL).

It is particularly important to develop strategies to reduce the burden of COPD in the early phase; i.e. early diagnosis [10] and collaborative management [11] in primary care. Most previous randomised trials were either hospital-based or enrolled patients with diverse degrees of severity, and considered a wide range of approaches. To our knowledge, only one previous comparative study specifically evaluated the LWWCOPD intervention with patients in primary care [12]. Therefore, the present study aimed to assess the effect of the LWWCOPD intervention on behavioural changes and disease-specific HRQoL in patients receiving primary care for COPD, compared to a control group of patients with COPD from a Dutch and Swiss primary care cohort (International collaborative effort on chronic obstructive lung disease-exacerbation risk index cohorts; ICE COLD ERIC).

\section{Methods}

\section{Study design}

This prospectively planned, non-randomised controlled study included patients with COPD from the mediX Zurich (Switzerland) primary care network that had participated in the LWWCOPD self-management programme from 2010 to 2012. The control group comprised patients with COPD who participated in the international ICE COLD ERIC cohort study, and who did not participate in the LWWCOPD programme (www.ClinicalTrials.gov NCT00706602) [13].

\section{Participants}

Inclusion criteria for both the mediX and ICE COLD ERIC cohorts were as follows: age $\geqslant 40$ years; a smoking habit (current or past) $\geqslant 10$ pack-years; a confirmed diagnosis of COPD; and a ratio of forced expiratory volume in $1 \mathrm{~s}$ to forced vital capacity (FEV1/FVC) $\leqslant 0.70$, with less than $12 \%$ and less than 200 $\mathrm{mL}$ increases in FEV1, post dilatation, according to the Global Initiative for Chronic Obstructive Lung Disease (GOLD) and GOLD II-IV classifications [14]. All participants were required to speak German, either as a native or "daily" language, and be able to attend training sessions for 6 weeks.

Patients with major psychiatric or terminal diseases were excluded. Of 148 eligible patients, 66 (45\%) were referred by general practitioners (GPs) from the network, and five were referred by the Zurich Lung Association. No information was available about the total number of patients invited and assessed by the Zurich Lung Association. Information on enrolment and exclusion was available for 35 of the $66(53 \%)$ patients referred by GPs. Of those 35 patients, two (6\%) were unwilling to participate, and two (6\%) had agendas that were incompatible with the programme. All referred patients had confirmed COPD; however, two showed features of a possible overlap of asthma and COPD, with a history of hay fever and childhood asthma.

The ICE COLD ERIC study was previously approved by all local ethics committees (Zürich EK-1519, St Gallen 08/065/1B, MEC 08/073 \# 08.17.0407). Additional approval for the present study was not required by the ethics committee (Zürich EK 50-2015).

\section{Intervention}

Patients in the mediX Zurich group received the LWWCOPD intervention [15] adapted for Swiss primary care [16]. Before implementation into primary care, starting in 2008, the LWWCOPD programme was conducted in the outpatient clinic of the University Hospital in Zurich. The experiences and learning needs identified for the improvement of COPD care, gained from secondary and tertiary care organisations, were considered in the plan for implementation of LWWCOPD into primary care. We followed the Plan, Do, Study, Act cycle [17]. 
The primary care LWWCOPD programme included three main dimensions: knowledge, skills and the confidence and motivation to use the skills. Emphasis was placed on the correct reactions to patient health deterioration, based on a written action plan. Therefore, an action plan was included as part of a personal diary. Patients were instructed to record the date and types of symptoms that worsened, and the action taken by the patient, according to the action plan. The action plan instructed patients to increase inhalation therapy, start systemic steroids and or antibiotics, contact the care team, seek clinical care and when necessary, seek hospital admission. We also emphasised the importance of regular physical activity, and strength, endurance and breathing exercises in the action plan. Participants were provided with the German version of the LWWCOPD book, which summarised the contents of the programme. The entire programme consisted of six group modules, including: 1) what is COPD; 2) pharmacological treatment and correct inhalation techniques; 3) breathing techniques and coping strategies aimed at symptom control; 4) how to manage daily activities/energy conservation; 5) the health benefits of physical activity and how to determine barriers and enablers of regular physical activity; and 6) what is an exacerbation and how to prevent, recognise and adequately manage worsening symptoms. Special attention was focused on "red flag" symptoms, like chest pain and/or acute severe dyspnoea. These were described as possible warning signs for acute heart problems or pulmonary embolism, and their occurrence required immediate contact with a doctor or hospital.

Patient beliefs and expectations were commented on, in an atmosphere of emphasised listening and concern, and personal experiences were discussed in a group setting. The aim was to instil confidence, enhance inner motivation to adopt healthy lifestyle behaviours, and empower patients to improve COPD self-management on a day-to-day basis. Programme adherence was assessed as the percentage of patients who participated in the assigned number of programme modules (maximum of six modules). After patient assessment and goal setting, modules were selected in consultation with the patient.

In addition to group sessions, each patient received two to three individual one-on-one coaching sessions to assess individual needs, goals, barriers and personal views and emotions that might affect their ability to cope and live with the disease. Strong emphasis was placed on the ability to use the personal written action plan correctly and safely. Confidence was supported by role-playing in various situations. Patients were invited to phone the care team when they had questions or concerns about starting emergency medications.

Two health professionals in the mediX group practice, a pulmonologist and a respiratory physiotherapist, conducted the entire programme. Both were trained and experienced in effective self-management support, motivational communication and interviewing. The respiratory physiotherapist was present at all modules to provide continuity and a connection between the patients and the care team. Feedback and collaboration with the treating physician was part of the programme. In addition, regular proactive follow-up calls were performed by a trained practice assistant at 3, 6, 12, 18 and 24 months, during which patients responded to lists of monitoring questions developed in cooperation with McGill University, Canada [18]. In addition, patients were able to contact the care team if they experienced deterioration.

Routine care comprised drug- and non-drug treatments for COPD, as indicated, to relieve symptoms and prevent exacerbations. We did not intervene in any way with treatment decisions from primary care physicians, or with patients in the ICE COLD ERIC cohort. Most patients were taking at least one long-acting bronchodilator, with or without an inhaled corticosteroid; about one third of the patients followed an exercise programme at home, or at a fitness or rehabilitation centre; and less than $2 \%$ of the patients followed a pulmonary rehabilitation programme in the year prior to enrolment [19].

\section{Primary and secondary outcomes}

The primary outcome was the HRQoL, measured with the Chronic Respiratory Questionnaire (CRQ) [20]. The minimal important difference (MID) was defined as a 0.5 -change, on a seven point scale, in a CRQ domain score [21]. The secondary outcome was the rate of moderate to severe exacerbations over a maximum of 2 years [22]. Assessments of self-reported exacerbations were prone to inaccuracy; therefore, we followed a central event adjudication process for both groups, as described previously [23]. Additional secondary outcomes included the smoking cessation rate and self-efficacy (only in the LWWCOPD group), assessed with an adapted Self-Efficacy for Managing Chronic Disease Scale [24].

\section{Statistical analyses}

To incorporate the longitudinal structure of the data, we used linear mixed models with random intercepts and random slopes to estimate between-group differences in the CRQ domains. To assess the total number of exacerbations that occurred over 2 years, we chose a zero-inflated negative binomial model to account for over-dispersion and the relatively high number of zeros in the data set. Smoking cessation was assessed 
among individuals that smoked at baseline; the logistic regression model was performed with an outcome variable of "continued smoking after 2 years".

Patients were not randomised in the present study. However, we performed a propensity score analysis. Thus, a logistic regression model was calculated for the outcome of "inclusion in the LWWCOPD programme". Based on model results, we calculated a propensity score (the predicted logarithmic probability of obtaining the treatment) for each person in the data set. Propensity scores were used to adjust all models to account for, as far as possible, the absence of randomisation. The logistic regression model included variables that influenced the propensity score, including sex, age, use of long-acting beta agonists (LABA) and/or inhaled corticosteroids (ICS), depression, FEV1, smoking at baseline, baseline CRQ dyspnoea score and a binary variable that indicated whether exacerbations had occurred within 12 months prior to starting the training programme. A separate propensity score was calculated for the models to analyse smoking cessation, due to the limited number of smokers at baseline.

The linear mixed models included the propensity score, the treatment variable (participation in the LWWCOPD programme) and a variable indicating the time point of the respective measurement (i.e. visits 1-4). To analyse the number of exacerbations, we used a two-part model; the negative binomial part of the model included only the propensity score and the treatment group; and the logistic part of the model included a binary variable indicating whether previous exacerbations had occurred. The propensity score and the treatment variable were also included in the logistic regression models for smoking cessation. In addition, we performed a sensitivity analysis that was restricted to controls that experienced an exacerbation in the previous year, for all outcomes, except smoking cessation; in the latter case, the new control group was too small for sufficient statistical power.

Analyses were conducted with the statistical programming language, $\mathrm{R}$ version 3.2.4 [25].

\section{Results}

\section{Group characteristics}

The study included 467 patients; 71 in the LWWCOPD self-management group and 396 in the ICE COLD ERIC routine care control group. At baseline, the LWWCOPD group showed a higher number of previous exacerbations, more severe airflow obstructions, and more frequent LABA/ICS treatments, but similar baseline HRQoL compared to the control group (table 1).

Effects on the primary outcome, HRQoL

Compared to the control group, the LWWCOPD group showed significant, clinically relevant (MID $>0.5$ ) treatment effects for all CRQ subscale scores, over the course of 1 year (table 2). The sensitivity analysis of

\begin{tabular}{lcc} 
TABLE 1 Patient characteristics at baseline & & \\
Characteristics & LWWCOPD group & ICE COLD ERIC group (control) \\
\hline Subjects $n$ & 71 & 396 \\
Age years & $69.3 \pm 10.3$ & $67.1 \pm 10.0$ \\
Male & $28(39.4 \%)$ & $225(56.8 \%)$ \\
CRQ mastery & $5.2 \pm 1.3$ & $5.5 \pm 1.2$ \\
CRQ dyspnoea & $4.6 \pm 1.2$ & $4.7 \pm 1.6$ \\
CRQ emotion & $4.9 \pm 1.2$ & $5.1 \pm 1.2$ \\
CRQ fatigue & $4.6 \pm 1.2$ & $4.4 \pm 1.4$ \\
Acute exacerbation of COPD in previous year & $51(71.8 \%)$ & $88(22.2 \%)$ \\
Smoking & $29(40.9 \%)$ & $164(41.4 \%)$ \\
Smoking history pack-years & $47.8 \pm 32.9$ & $44.5 \pm 27.8$ \\
FEV $\%$ & $52.4 \pm 17.6$ & $55.9 \pm 16.5$ \\
Comorbidities & & $115(29.9 \%)$ \\
Cardiovascular & $29(40.8 \%)$ & $57(14.8 \%)$ \\
Diabetes & $8(11.2 \%)$ & $23(6.0 \%)$ \\
Depression & $14(19.7 \%)$ & $260(65.7 \%)$ \\
LABA/ICS & $62(87.3 \%)$ & $112(28.3 \%)$ \\
Exercise programme & $20(28.2 \%)$ & $6(1.5 \%)$ \\
Pulmonary rehabilitation in the previous year & $1(1.4 \%)$ &
\end{tabular}

Data presented as mean \pm SD, unless otherwise indicated. COPD: chronic obstructive pulmonary disease; LWWCOPD: Living well with COPD; ICE COLD ERIC: International collaborative effort on chronic obstructive lung disease-exacerbation risk index cohorts; CRQ: Chronic Respiratory Questionnaire; FEV1: forced expiratory volume in $1 \mathrm{~s}$; LABA/ICS: long-acting beta agonists/inhaled corticosteroids. 
TABLE 2 Comparison of health-related quality of life changes (Chronic Respiratory Questionnaire domains) and disease exacerbations (unscheduled visits and hospitalisations) between the intervention and control groups

\begin{tabular}{lcc} 
Variable & Group difference (95\% CI) & p-value \\
\hline Mastery & $0.54(0.13-0.94)$ & 0.010 \\
Fatigue & $0.55(0.11-0.99)$ & 0.014 \\
Emotional function & $0.54(0.14-0.93)$ & 0.008 \\
Dyspnoea & $0.64(0.14-1.14)$ & 0.010 \\
Incidence rate ratio of exacerbations (moderate and severe) & $0.36(0.25-0.52)$ & $<0.0001$ \\
\hline
\end{tabular}

control patients with previous exacerbations showed similar overall results, except for slightly greater treatment effects on fatigue $(0.71,95 \%$ CI 0.13 to 1.29$)$ and dyspnoea (0.72, 95\% CI 0.14 to 1.31$)$; and slightly smaller effects on mastery $(0.44,95 \% \mathrm{CI}-0.06$ to 0.95$)$ and emotional function $(0.47,95 \% \mathrm{CI}$ -0.01 to 0.95 ).

\section{Effect on exacerbations}

The LWWCOPD group had considerably fewer moderate to severe exacerbations over 24 months (mean number of exacerbations 1.31 , SD 1.55, range 0 to 7 ), compared to the control group (mean \pm SD number of exacerbations $1.59 \pm 2.11$, range $0-12$ ). The exacerbation incidence rate ratio (IRR) was 0.36 (95\% CI 0.25 to 0.52 ). The sensitivity analysis showed similar results (IRR $0.41,95 \%$ CI 0.26 to 0.65 ).

\section{Effect on smoking cessation}

Odds ratios for patients that continued smoking at 1 and 2 years were 1.06 (95\% CI 0.31 to 4.03 ) and 0.65 (95\% CI 0.2 to 2.22), respectively, in the LWWCOPD group.

\section{Adherence to the LWWCOPD programme}

Every patient in the LWWCOPD group was expected to participate in six modules. However, it was possible to join only those modules that were considered essential, after patients were assessed and individual goals were set. All but six patients (92\%) were offered all six modules, and 59 participated in all assigned modules, which indicated high adherence (90\%).

\section{Effect on self-efficacy for intended health behaviour}

Significant increases from baseline to 2 years were observed in patient confidence in recognising deterioration at an early stage $(\mathrm{p}<0.001)$, performing the correct inhalation technique $(\mathrm{p}<0.001)$, and confidence in the timely and correct use of the action plan (table 3). The confidence in managing to be physically active for 30 min twice per week showed the largest variability of all measurements at baseline. It increased over the first 6 months of the intervention $(p<0.001)$, then started to decrease, and then increased again after 1 year, and returned to baseline after 2 years.

10 patients actively asked for a prescription and started a pulmonary rehabilitation programme. Seven patients purchased step counters; five started physical activity in fitness centres; five purchased home-trainers; one bought an e-bike; and one started power plate training.

During the study period, six patients in the intervention group (8.4\%) and 38 patients in the control group (9.6\%) died.

\section{Discussion}

This study showed that the LWWCOPD intervention in a primary care setting improved HRQoL to a clinically relevant extent and reduced COPD exacerbations.

The intervention mainly comprised motivational and cognitive behavioural components, which emphasised self-efficacy and the tools to become a "successful self-manager". Some factors were critical to success. First, the care team, based on the primary care setting, comprised a respiratory physiotherapist, pulmonologist and trained practice assistants. This team structure carried the advantages of care continuity, familiarity and communication compared to external specialised settings. Another key element to success was regular contact, which empowered patients and promoted safety. The practice assistants received safety training as part of the patient coaching training. 
TABLE 3 Self-efficacy over time for chronic obstructive pulmonary disease behaviour linked to the intervention

\begin{tabular}{lcccc} 
Confidence in $^{\#}$ & $\begin{array}{c}\text { Baseline } \\
\text { median (IQR) }\end{array}$ & $\begin{array}{c}\text { 3-month } \\
\text { median (IQR) }\end{array}$ & $\begin{array}{c}\text { 6-month } \\
\text { median (IQR) }\end{array}$ & $\begin{array}{c}\text { 24-month } \\
\text { median (IQR) }\end{array}$ \\
\hline Daily inhalation & $10(9-10)$ & $10(10-10)$ & $10(10-10)$ & $10(10-10)$ \\
Correct inhalation technique & $8(8-10)$ & $10(10-10)$ & $10(10-10)$ & $10(10-10)$ \\
Timely recognition of deterioration & $7(5-8)$ & $10(9-10)$ & $10(9-10)$ & $10(10-10)$ \\
Timely use of the action plan 7 & & $10(9-10)$ & $10(9-10)$ & $10(8-10)$ \\
Using the action plan correctly & & $10(9-10)$ & $10(10-10)$ & $10(9-10)$ \\
Managing to be physically active for & $7(4-10)$ & $10(9-10)$ & $10(8-10)$ & $7(5-10)$ \\
$\quad 30$ min twice per week & & & & \\
\end{tabular}

IQR: interquartile range. " : rated on a scale of 0 (not at all confident) to 10 (totally confident); ${ }^{\text {I }}$ : no patient had an action plan at baseline.

Our results also demonstrated the effectiveness of adequate self-management on health outcomes [26] arising from improved self-efficacy, mastery and integration of self-management skills on a day-to-day basis [4, 7]. Patients in the LWWCOPD group gained confidence in recognising early signs of deterioration and using the action plan in a timely fashion, which improved exacerbation outcomes, consistent with previous studies [27-29]. However, confidence in the timely use of the action plan declined in later stages, which emphasised the need for regular follow-up and proactive care.

The confidence in being physically active increased during the first 6 months of the intervention. Indeed, physical activity increased in $57 \%$ of the intervention group. This window of opportunity, when patients were highly motivated to increase their physical activity levels, represented the optimal time to support them in finding and starting their preferred field of physical activity, encourage them to attend an outpatient pulmonary rehabilitation programme and motivate them to continue those activities. BENZO et al. [9] also reported that pulmonary rehabilitation attendance was higher $(>17 \%)$ in a coached group than in a control group. Those results suggested that pulmonary rehabilitation practices might benefit from switching the primary emphasis from physical training and some education to self-management coaching as initial point.

We found that in a "real-world" primary care setting, patients showed improvements that were comparable to, or better than, those reported in systematic reviews [30-32] and in a recent Cochrane review on COPD self-management interventions with an exacerbation action plan [33]. An earlier comparative study on the LWWCOPD intervention in a primary care setting [12] could not confirm the effect reported in the original Canadian trial [15]. Potential explanations for that discrepancy could be the lower programme intensity and lack of group coaching. In contrast, we emphasised patient motivation and self-efficacy, with role modelling and interactions in group sessions.

BENZO et al. [9] found that health coaching improved emotional function, based on a somewhat unusual CRQ domain of mastery and emotional function. This improvement was considered a potentially "ground-breaking finding", owing to the knowledge gap that exists regarding COPD interventions that improve emotions. Improved emotional function, combined with increased self-efficacy in recognising and managing exacerbations, might account for our results on unscheduled visits and hospitalisations.

Although we did not perform one, a former cost-utility analysis showed that the LWWCOPD intervention saved costs [34]. Considering that our intervention significantly reduced the risk of exacerbations, we could expect economic benefits, due to the reductions in patient caseloads and hospitalisations.

The main strengths of this study were its prospectively planned controlled design in a primary care setting and the coordinated data collection between the mediX and ICE COLD ERIC cohorts, which allowed controls for the confounders. Another strength was that we accurately described the concept and components of the intervention. Moreover, our 2-year follow-up was sufficiently long to demonstrate the effects of LWWCOPD on the exacerbation rate, an important complication, prognostic factor and cost driver in COPD.

The main limitation of this study was the non-randomised design. Based on the available evidence and internal and external validity issues, we carefully weighed the advantages of a randomised controlled trial compared to a prospectively planned comparative study. We decided that the latter design was better suited for bridging an important gap in the evidence base. Indeed, based on existing evidence [33], another randomised trial demonstrated that intervention efficacy would not have provided any crucial 
information (and might not have been justified). Instead, we aimed to assess whether an intervention based on proven self-management methods was feasible and effective in a real-world primary care COPD population. This approach was only possible by enrolling consecutive patients that followed a self-management programme. In contrast, a randomised controlled trial would exclude a substantial proportion of patients, owing to a failure to obtain consent for a random assignment to intervention or control treatment (a common challenge in non-pharmacological interventions), or due to restrictive eligibility criteria. In addition to maximising external validity, we ensured internal validity with a prospective study plan, combined with sensitivity analysis that was restricted to control patients with previous exacerbations.

Future head-to-head randomised controlled trials with well-described self-management interventions that aim to change behaviour should determine the degrees of behavioural changes effected (particularly in the long-term) by different approaches. In addition, long-term follow-up studies might identify patient characteristics that indicate the ability to adopt adequate self-management skills into daily life and those that indicate a need for ongoing professional support.

\section{Conclusion and implications}

This study showed that the LWWCOPD intervention in primary care effectively improved disease coping skills and HRQoL and reduced the risk of exacerbations in patients with COPD. Our results might not be generalisable to all primary care practices; however, they might motivate transformation and pave the way for better support and training of primary care teams. This strategy will contribute to delivering high quality COPD care and effective chronic disease management.

\section{Acknowledgements}

We thank Jean Bourbeau, McGill University, Canada, for his valuable support, cooperation and helpful discussions in adapting the LWWCOPD programme and instruments to a Swiss primary care setting (http://www.livingwellwithcopd. com). We are grateful to Felix Huber, Valeria Maissen, Simone Stoller and Angela Buschor from the mediX group practice, for their willingness and ongoing support in transforming the delivery of care to patients with COPD, and thus, making it feasible to implement the LWWCOPD intervention. We thank Lunge Zürich, a non-profit community organisation. In particular, we thank Sandra Catuogno-Brülisauer and Alex Turk, who assisted in the adaptation and production of LWWCOPD materials for Switzerland. Finally, we thank all patients and GPs that participated in this study.

Author contributions: C. Steurer-Stey, K. Dalla Lana and M.A. Puhan initiated the Swiss adaptation of the Canadian LWWCOPD and its implementation into primary care. C. Steurer-Stey and K. Dalla Lana constructed, organised and taught the LWWCOPD intervention within the mediX group practice. G. ter Riet coordinated ICECOLD ERIC in the Netherlands. M.A. Puhan, C. Steurer-Stey, K. Dalla Lana and G. ter Riet formulated the concept and questions to be addressed by this study. J. Braun performed the statistical analyses. All authors agreed on the initial outline of this manuscript, reviewed it, provided comments on each draft and approved the final version.

\section{References}

1 Wagner EH, Austin BT, Von Korff M. Organizing care for patients with chronic illness. Milbank Q 1996; 74: 511-544.

2 Ellrodt G, Cook DJ, Lee J, et al. Evidence-based disease management. JAMA 1997; 278: 1687-1692.

3 Fromer L. Implementing chronic care for COPD: planned visits, care coordination, and patient empowerment for improved outcomes. Int J Chron Obstruct Pulmon Dis 2011; 6: 605-614.

4 Lorig KR, Holman H. Self-management education: history, definition, outcomes, and mechanisms. Ann Behav Med 2003; 26: 1-7.

5 Barlow J, Wright C, Sheasby J, et al. Self-management approaches for people with chronic conditions: a review. Patient Educ Couns 2002; 48: 177-187.

6 Kaptein AA, Scharloo M, Fischer MJ, et al. 50 years of psychological research on patients with COPD--road to ruin or highway to heaven? Respir Med 2009; 103: 3-11.

7 Kaptein AA, Fischer MJ, Scharloo M. Self-management in patients with COPD: theoretical context, content, outcomes, and integration into clinical care. Int J Chron Obstruct Pulmon Dis 2014; 9: 907-917.

8 Huber M, Knottnerus JA, Green L, et al. How should we define health? BMJ 2011; 343: d4163.

9 Benzo R, Vickers K, Novotny PJ, et al. Health coaching and chronic obstructive pulmonary disease rehospitalization. A randomized study. Am J Respir Crit Care Med 2016; 194: 672-680.

10 Jordan RE, Jowett S, Fitzmaurice D, et al. COPD case finding: effective, but also cost-effective? - Authors' reply. Lancet Respir Med 2016; 4: e50.

11 Foot H, Freeman C, Hemming K, et al. Reducing Medical Admissions into Hospital through Optimising Medicines (REMAIN HOME) Study: protocol for a stepped-wedge, cluster-randomised trial. BMJ Open 2017; 7: e015301.

12 Bischoff EW, Akkermans R, Bourbeau J, et al. Comprehensive self management and routine monitoring in chronic obstructive pulmonary disease patients in general practice: randomised controlled trial. BMJ 2012; 345: e7642.

13 Siebeling L, ter Riet G, van der Wal WM, et al. ICE COLD ERIC - International collaborative effort on chronic obstructive lung disease: exacerbation risk index cohorts - study protocol for an international COPD cohort study. BMC Pulm Med 2009; 9: 15. 
14 Global Strategy for the Diagnosis Management, and Prevention of COPD. Global Initiative for Chronic Obstructive Lung Disease (GOLD), 2015. Available from: www.goldcopd.org

15 Bourbeau J, Julien M, Maltais F, et al. Reduction of hospital utilization in patients with chronic obstructive pulmonary disease: a disease-specific self-management intervention. Arch Intern Med 2003; 163: 585-591.

16 Steurer-Stey C, DallaLana K, Stoller S, et al. Rethinking models of care to improve chronic care in COPD patients. Respiration (Basel) 2011; 82: 80.

17 Berwick DM, Nolan TW. Physicians as leaders in improving health care: a new series in Annals of Internal Medicine. Ann Intern Med 1998; 128: 289-292.

18 Living well with Chronic Obstructive Pulmonary Disease. www.livingwellwithcopd.com Date last updated: July 4, 2016.

19 Siebeling L, Puhan MA, Muggensturm P, et al. Characteristics of Dutch and Swiss primary care COPD patients baseline data of the ICE COLD ERIC study. Clin Epidemiol 2011; 3: 273-283.

20 Puhan MA, Behnke M, Frey M, et al. Self-administration and interviewer-administration of the German Chronic Respiratory Questionnaire: instrument development and assessment of validity and reliability in two randomised studies. Health Qual Life Outcomes 2004; 2: 1.

21 Schunemann HJ, Puhan M, Goldstein R, et al. Measurement properties and interpretability of the Chronic respiratory disease questionnaire (CRQ). COPD 2005; 2: 81-89.

22 Rodriguez-Roisin R. Toward a consensus definition for COPD exacerbations. Chest 2000; 117: Suppl. 2, 398S-401S.

23 Frei A, Siebeling L, Wolters C, et al. The Inaccuracy of Patient Recall for COPD exacerbation rate estimation and its implications: results from central adjudication. Chest 2016; 150: 860-868.

24 Lorig KR, Sobel DS, Ritter PL, et al. Effect of a self-management program on patients with chronic disease. Eff Clin Pract 2001; 4: 256-262.

25 R Core Team. R: A language and environment for statistical computing. R.Foundation for Statistical Computing. 2016. Available from: www.r-project.org

26 Bourbeau J, Nault D, Dang-Tan T. Self-management and behaviour modification in COPD. Patient Educ Couns 2004; 52: 271-277.

27 Wilkinson TM, Donaldson GC, Hurst JR, et al. Early therapy improves outcomes of exacerbations of chronic obstructive pulmonary disease. Am J Respir Crit Care Med 2004; 169: 1298-1303.

28 Bischoff EW, Hamd DH, Sedeno M, et al. Effects of written action plan adherence on COPD exacerbation recovery. Thorax 2011; 66: 26-31.

29 Bucknall CE, Miller G, Lloyd SM, et al. Glasgow supported self-management trial (GSuST) for patients with moderate to severe COPD: randomised controlled trial. BMJ 2012; 344: e1060.

30 Kruis AL, Smidt N, Assendelft WJ, et al. Integrated disease management interventions for patients with chronic obstructive pulmonary disease. Cochrane Database Syst Rev 2013; CD009437.

31 Zwerink M, Brusse-Keizer M, van der Valk PD, et al. Self management for patients with chronic obstructive pulmonary disease. Cochrane Database Syst Rev 2014; CD002990.

32 Adams SG, Smith PK, Allan PF, et al. Systematic review of the chronic care model in chronic obstructive pulmonary disease prevention and management. Arch Intern Med 2007; 167: 551-561.

33 Lenferink A, Brusse-Keizer M, van der Valk PD, et al. Self-management interventions including action plans for exacerbations versus usual care in patients with chronic obstructive pulmonary disease. Cochrane Database Syst Rev 2017; CD011682.

34 Bourbeau J, Collet JP, Schwartzman K, et al. Economic benefits of self-management education in COPD. Chest 2006; 130: 1704-1711. 